\title{
Nonlinear Robust Control of a Hypersonic Flight Vehicle Using Fuzzy Disturbance Observer
}

\author{
Lei Zhengdong, ${ }^{1}$ Wang Man, ${ }^{1}$ and Yang Jianying ${ }^{2}$ \\ ${ }^{1}$ College of Engineering, Peking University, Beijing 100871, China \\ ${ }^{2}$ State Key Laboratory for Turbulence and Complex Systems, Department of Mechanics and Aerospace Engineering, Peking University, \\ Beijing 100871, China
}

Correspondence should be addressed to Yang Jianying; yjy@pku.edu.cn

Received 27 May 2013; Accepted 5 September 2013

Academic Editor: Hongli Dong

Copyright (C) 2013 Lei Zhengdong et al. This is an open access article distributed under the Creative Commons Attribution License, which permits unrestricted use, distribution, and reproduction in any medium, provided the original work is properly cited.

\begin{abstract}
This paper is concerned with a novel tracking controller design for a hypersonic flight vehicle in complex and volatile environment. The attitude control model is challengingly constructed with multivariate uncertainties and external disturbances, such as structure dynamic and stochastic wind disturbance. In order to resist the influence of uncertainties and disturbances on the flight control system, nonlinear disturbance observer is introduced to estimate them. Moreover, for the sake of high accuracy and sensitivity, fuzzy theory is adopted to improve the performance of the nonlinear disturbance observer. After the total disturbance is eliminated by dynamic inversion method, a cascade system is obtained and then stabilized by a sliding-mode controller. Finally, simulation results show that the strong robust controller achieves excellent performance when the closed-loop control system is influenced by mass uncertainties and external disturbances.
\end{abstract}

\section{Introduction}

The study of the hypersonic flight vehicle (HFV) has been implemented for more than two decades all around the world. As early as 1980s, Chavez and Schmidt had made pivotal works of developing classic longitudinal model of the vehicle dynamics $[1,2]$. Afterwards, Bolender and Doman developed the important implicit model: the first-principle model in [3]. In recent years, flexible effect, which results in structure dynamic and may significantly influence the performance of HFV, attracts more and more attention. Some models introduce the flexible modes in the motion equations $[4,5]$. The most significant characteristics of HFV are the highly coupled and nonlinear nature of its dynamic behavior. Additionally, complicated flight condition and variability of the vehicle characteristics bring about mass uncertainties for the modeling. Some researchers, such as McNamara and Friedmann, have stated the difficulties faced in the modeling and control in their works [6]. At present, the models are unprecedented, complex, and much closer to the reality.

A large amount of different control methods has been successfully applied to stabilize the system of HFV and accomplish special tasks. Some typical approaches can be much weighted, such as linear parameter-varying control [7], adaptive control $[8,9]$, sliding-mode control [10], gainscheduling, dynamic inversion [11], and $\mathscr{H}_{\infty}$ design [12]. Some other control methods (e.g., linear matrix inequity (LMI)) are based on linearized model by using the small disturbance principle at given trim point [13]. However, most of these methods have much conservation because the controller should be redesigned if the trim points of the system are altered and the calculation is amazingly tremendous. Thus, nonlinear control methods, such as dynamic inversion and adaptive control, seem more effective and present good relationship with physical process.

Despite of recent success of NASA's X-51A experiment, the design of robust guidance and control systems for HFV is still a challenging area [14]. Due to the complex flight environment (e.g., high mach, low air density, and thermalelasticity), seeking for a strong robust controller is on edge. This kind of controller cannot only adapt to severe environment but also tolerate its own faults. As for HFV, one of the most important tasks is to track the reference trajectory. However, some unknown factors, such as gust wind, 
structure dynamic, actuator failure, and sensor fault, bring about great uncertainties to the system performance. Some linearized models have been stabilized by robust controller based on LMI, in which Lyapunov stability theory is widely applied [15]. In [16], energy norm indices have been used for the fault detection problem in discrete-time Markovian jump systems. Some other novel methods also successfully addressed the uncertainties and disturbance problems, such as in [17]; it used extended Kalman filter to solve the problems of stochastic nonlinearities and multiple missing measurements instead of using observing methods. Nevertheless, in $[13,18]$, the parameters and states in linearized models are all the deviations from the trim point rather than the real ones. Thus, nonlinear analysis methods may be more meaningful in the practical engineering. The study of faulttolerant control, which is based on nonlinear observer, makes great contributions to the design of strong robust controller. There are lots of various state estimators at present. Such as in $[19,20]$, state estimators were constructed with data from sensor networks for discrete-time systems to resist bad influence by sensor saturation, delay, and uncertainties. In [14], a fuzzy disturbance observer was proposed to estimate the uncertainties and disturbances. The nonlinear observer cannot only reconfigure the original states but also monitor the total disturbance. Then, the disturbance can be eliminated by using dynamic inverse method. As for this method, descriptions for disturbance and uncertainties are more accurate and rational for the reality.

In order to achieve excellent performance for the disturbance observer, in this paper, we introduce a novel fuzzy disturbance observer (FDO) to monitor the total disturbance, which can intelligently change its behavior according to the error of observation. What we contribute is that we redefine the disturbance as the sum of internal disturbance and external disturbance. This conception was first proposed in active disturbance rejection control (ADRC) by Han [21]. This redefinition reduces the quantity of calculation compared to other online methods, such as adaptive control. Moreover, it becomes feasible to apply this technology to electronic programming [21].

In this paper, our control objective is a kind of HFV similar to High-altitude Test Vehicle-2 (HTV-2). The goal for control is to make the attitude states (angle of attack, angle of the sideslip, and angle of roll) track the reference trajectories as soon as possible, especially when the flight environment contains large scale of uncertainties and disturbances. The rest of the paper is arranged as follows. Section 2 demonstrates the attitude model of the flight vehicle with mass uncertainties in detail. And the disturbance observer based on fuzzy theory is introduced in Section 3. In Section 4, controller is designed based on the disturbance observer. Simulation results in Section 5 confirm the controller's excellent operation and how well the observer monitors the disturbance in real time.

\section{Nonlinear Attitude Control Model}

2.1. Establishment of Nonlinear Dynamic Model. Some researchers have made great contributions to the modeling for HFV. HFV is usually characterized with strong coupling, strong nonlinear and multiple inputs and multiple outputs (MIMO). It is nearly impossible to do research on a highly accurate model considering all aspects. Thus, tradeoff between the modeling accuracy and simplicity should be considered. According to Newton's second law of motion and three-dimension coordinate transformation, the model of attitude motions for the MIMO flight vehicle model with two inputs $\left(\delta_{r}\right.$ and $\left.\delta_{l}\right)$ can be established.

The angle equations are listed as follows:

$$
\begin{gathered}
\dot{\alpha}=\omega_{z}+\frac{\sin \alpha \sin \beta}{\cos \beta} \omega_{y}-\frac{\cos \alpha \sin \beta}{\cos \beta} \omega_{x} \\
-\frac{\left(-C_{A} q S-m g \sin \phi_{r} \cos \psi_{r}\right) \sin \alpha}{m V_{r} \cos \beta} \\
-\left(\left(C_{N} q S-m g \cos \phi_{r} \cos \gamma\right.\right. \\
\dot{\beta}=\omega_{y} \cos \alpha+\omega_{x} \sin \alpha \\
+\frac{\left.C_{N} q S-m g\left(\cos \phi_{r} \cos \gamma+\sin \phi_{r} \sin \psi_{r} \sin \gamma\right) \cos \alpha\right)}{\left.m V_{r} \sin \gamma\right)} \\
\times \sin \alpha \sin \beta \\
\quad \frac{-C_{A} q S-m g \sin \phi_{r} \cos \psi_{r}}{m V_{r}} \cos \alpha \sin \beta \\
\dot{\gamma}=\omega_{x}+\dot{\phi}_{r} \sin \psi_{r} .
\end{gathered}
$$

And the angle-rate equations are given as

$$
\begin{aligned}
& J_{x} \dot{\omega}_{x}-J_{x y} \dot{\omega}_{y}-J_{x z} \dot{\omega}_{z}+J_{z} \omega_{y} \omega_{z}-J_{x z} \omega_{x} \omega_{y} \\
&-J_{y z} \omega_{y}^{2}-J_{y} \omega_{y} \omega_{z}+J_{x y} \omega_{x} \omega_{z}+J_{y z} \omega_{z}^{2} \\
&= C_{l} q S L-\frac{C_{l p} q S L^{2} \omega_{x}}{V_{r}}, \\
& J_{y} \dot{\omega}_{y}-J_{x y} \dot{\omega}_{x}-J_{y z} \dot{\omega}_{z}+J_{x} \omega_{x} \omega_{z}-J_{x y} \omega_{y} \omega_{z} \\
& \quad-J_{x z} \omega_{z}^{2}-J_{z} \omega_{x} \omega_{z}+J_{x z} \omega_{x}^{2}+J_{y z} \omega_{x} \omega_{y} \\
&=C_{n} q S L-\frac{C_{n r} q S L^{2} \omega_{y}}{V_{r}}, \\
& J_{z} \dot{\omega}_{z}-J_{x z} \dot{\omega}_{x}-J_{y z} \dot{\omega}_{y}+J_{y} \omega_{x} \omega_{y}-J_{x y} \omega_{x}^{2} \\
&-J_{y z} \omega_{x} \omega_{z}-J_{x} \omega_{x} \omega_{y}+J_{x y} \omega_{y}^{2}+J_{x z} \omega_{y} \omega_{z} \\
&=C_{m} q S L-\frac{C_{m q} q S L^{2} \omega_{z}}{V_{r}},
\end{aligned}
$$


where $C_{A}, C_{N}, C_{Z}, C_{l}, C_{m}, C_{n}, C_{l p}, C_{m q}$, and $C_{n r}$ are related to one or four parameters (their expressions are listed below) and can be gotten by interpolation method:

$$
\begin{gathered}
C_{i}\left(\alpha, \beta, \delta_{r}, \delta_{l}\right)(i=A, N, Z, l, m, n), \\
C_{j}(\alpha)(j=l p, m q, n r) .
\end{gathered}
$$

Figure 1 shows the moment and force curves and their fitted functions versus $\alpha$ and $\beta$, respectively. As the coefficients are determined by four variables: $\alpha, \beta, \delta_{r}$, and $\delta_{l}$. The inputs $\delta_{r}$ and $\delta_{l}$ are both frozen to 0.1 degree to draw the threedimensional pictures below.

Therefore, (1) and (2) can be rewritten to (4) by separation of variables:

$$
\begin{gathered}
\dot{\Theta}=\mathbf{f}_{1}(\boldsymbol{\Theta})+\mathbf{M}(\boldsymbol{\Theta}) \boldsymbol{\Omega} \\
\dot{\boldsymbol{\Omega}}=\mathbf{f}_{2}(\boldsymbol{\Theta}, \boldsymbol{\Omega})+\mathbf{B}(\boldsymbol{\Theta}) \mathbf{u},
\end{gathered}
$$

where

$$
\begin{gathered}
\boldsymbol{\Theta}=\left[\begin{array}{lll}
\alpha & \beta & \gamma
\end{array}\right]^{T}, \quad \boldsymbol{\mathbf { u }}=\left[\begin{array}{lll}
\delta_{r} & \delta_{l}
\end{array}\right]^{T}, \\
\mathbf{M}(\boldsymbol{\Theta})=\left[\begin{array}{ccc}
-\cos \alpha \tan \beta & \sin \alpha \tan \beta & 1 \\
\sin \alpha & \cos \alpha & 0 \\
1 & 0 & 0
\end{array}\right], \\
\mathbf{B}(\boldsymbol{\Theta})=\left[\begin{array}{ccc}
J_{x} & -J_{x y} & -J_{x z} \\
-J_{x y} & J_{y} & -J_{y z} \\
-J_{x z} & -J_{y z} & J_{z}
\end{array}\right]^{-1}\left[\begin{array}{cc}
C_{C_{l}}^{\delta_{r}} & C_{C_{l}}^{\delta_{l}} \\
C_{C_{n}}^{\delta_{r}} & C_{C_{n}}^{\delta_{l}} \\
C_{C_{m}}^{\delta_{r}} & C_{C_{m}}^{\delta_{l}}
\end{array}\right] .
\end{gathered}
$$

Equation (4) is a typical system that can be stabilized by back-stepping method, in which $\Omega$ can be seen as a pseudoinput. In this system, $V_{r}, \phi_{r}$, and $\psi_{r}$ are all determined by the reference trajectory and known for controller design.

2.2. Problem Formulation. As for describing the complex and volatile flight condition, a great amount of parameter uncertainties and stochastic wind disturbance is introduced into this model. The parameter uncertainties and external disturbance (bandlimited and varying slowly) are expressed in the equations as follows:

$$
\begin{gathered}
\dot{\boldsymbol{\Theta}}=\left(\mathbf{f}_{1}(\boldsymbol{\Theta})+\Delta \mathbf{f}_{1}(\boldsymbol{\Theta})\right)+(\mathbf{M}(\boldsymbol{\Theta})+\Delta \mathbf{M}(\boldsymbol{\Theta})) \boldsymbol{\Omega}+\mathbf{d}_{\text {wind } 1}, \\
\dot{\boldsymbol{\Omega}}=\left(\mathbf{f}_{2}(\boldsymbol{\Theta}, \boldsymbol{\Omega})+\Delta \mathbf{f}_{2}(\boldsymbol{\Theta}, \boldsymbol{\Omega})\right)+(\mathbf{B}(\boldsymbol{\Theta})+\Delta \mathbf{B}(\boldsymbol{\Theta})) \mathbf{u}+\mathbf{d}_{\text {wind } 2} .
\end{gathered}
$$

In system (6), $\mathbf{f}_{1}$ and $\mathbf{f}_{2}$ contain lots of complicated structure information of this model, which are too complicated if they are written into formula expression. For simplicity, some items are rewritten as the following expression:

$$
\begin{gathered}
\boldsymbol{\Pi}_{1}(\boldsymbol{\Theta}, \boldsymbol{\Omega})=\mathbf{f}_{1}(\boldsymbol{\Theta})+\Delta \mathbf{f}_{1}(\boldsymbol{\Theta})+\Delta \mathbf{M}(\boldsymbol{\Theta}) \boldsymbol{\Omega}+\mathbf{d}_{\text {wind }_{1}}, \\
\boldsymbol{\Pi}_{2}(\boldsymbol{\Theta}, \boldsymbol{\Omega})=\mathbf{f}_{2}(\boldsymbol{\Theta}, \boldsymbol{\Omega})+\Delta \mathbf{f}_{2}(\boldsymbol{\Theta}, \boldsymbol{\Omega})+\Delta \mathbf{B}(\boldsymbol{\Theta}) \mathbf{u}+\mathbf{d}_{\text {wind }_{2}},
\end{gathered}
$$

thus, system (6) can be rewritten as

$$
\dot{\Gamma}=\mathbf{K}(\Theta) \mathbf{U}+\Pi(\Theta, \Omega),
$$

where

$$
\begin{array}{cc}
\Gamma=\left[\begin{array}{l}
\Theta \\
\boldsymbol{\Omega}
\end{array}\right], & \mathbf{U}=\left[\begin{array}{l}
\boldsymbol{\Omega} \\
\mathbf{u}
\end{array}\right], \\
\mathbf{K}(\boldsymbol{\Theta})=\left[\begin{array}{cc}
\mathbf{M}(\boldsymbol{\Theta}) & \mathbf{0} \\
\mathbf{0} & \mathbf{B}(\boldsymbol{\Omega})
\end{array}\right], & \boldsymbol{\Pi}(\boldsymbol{\Theta}, \boldsymbol{\Omega})=\left[\begin{array}{l}
\Pi_{1}(\boldsymbol{\Theta}, \boldsymbol{\Omega}) \\
\Pi_{2}(\boldsymbol{\Theta}, \boldsymbol{\Omega})
\end{array}\right] .
\end{array}
$$

In particular, total disturbance is redefined as $\boldsymbol{\Pi}_{i}(\boldsymbol{\Theta}, \boldsymbol{\Omega})(i=1,2) \in \mathbf{R}^{3 \times 1}$, unknown and bandlimited, to represent the sum of partial structure information, model parameter uncertainties, and wind disturbance. From the above consideration, a cascade system with total disturbance is presented. As for the practical hypothesis, the total disturbance, hence, contains wide range of parameter uncertainties, such as force and moment coefficients deviation, inertia deviation, gravity center deviation, and atmosphere density deviation. As the goal for flight control, the goal is to converge the flight attitude and angle-rate $(\boldsymbol{\Theta}(t), \boldsymbol{\Omega}(t))$ into the given reference trajectory $\left(\boldsymbol{\Theta}^{*}(t), \boldsymbol{\Omega}^{*}(t)\right)$ in finite time. And the transient response performance should be as better as the system can achieve. Consider the following:

$$
\begin{aligned}
& \lim _{t \rightarrow+\infty}\left\|\boldsymbol{\Theta}(t)-\boldsymbol{\Theta}^{*}(t)\right\|=\mathbf{0}, \\
& \lim _{t \rightarrow+\infty}\left\|\boldsymbol{\Omega}(t)-\boldsymbol{\Omega}^{*}(t)\right\|=\mathbf{0} .
\end{aligned}
$$

Dynamic inversion method has been widely used to address such problem; however, the most critical and difficult work is the disturbance estimation. Disturbance estimation relies on identification via inputs and outputs data. As long as the total disturbance is estimated (notated as $\widehat{\Pi}_{i}(i=1,2)$ ), the problem surely becomes easy to disentangle by dynamic inversion method as the following expression; $\boldsymbol{\Omega}$ behaves as a pseudoinput: $\boldsymbol{\Omega}_{c}$. Consider the following:

$$
\begin{aligned}
\boldsymbol{\Omega}_{c} & =\mathbf{M}^{-1}(\boldsymbol{\Theta})\left(-\widehat{\boldsymbol{\Pi}}_{1}+\boldsymbol{\Omega}\right), \\
\mathbf{u} & =\mathbf{B}^{\dagger}(\boldsymbol{\Theta})\left(-\widehat{\boldsymbol{\Pi}}_{2}+\mathbf{u}_{c}\right) .
\end{aligned}
$$

In (11), the inversion matrix $\mathbf{B}^{-1}$ cannot be obtained because $\mathbf{B}$ is not a square matrix. Thus, pseudoinversion $\mathbf{B}^{\dagger}$ is used to replace it, and the error which results from inaccurate dynamic inversion can be reduced by error-integral feedback. If $\widehat{\Pi}_{i}$ can be perfectly monitored, the plant system (4) can be rewritten as, by replacing with (11),

$$
\begin{aligned}
& \dot{\boldsymbol{\Theta}}=\boldsymbol{\Omega}+\boldsymbol{\Pi}_{1}-\widehat{\boldsymbol{\Pi}}_{1}, \\
& \dot{\boldsymbol{\Omega}}=\mathbf{u}_{c}+\boldsymbol{\Pi}_{2}-\widehat{\boldsymbol{\Pi}}_{2} .
\end{aligned}
$$

Assumption: the observation error $\left(\widehat{\Pi}_{i}-\Pi_{i}\right)$ is minor, and its effect on the stability of the closed loop system is negligible. This assumption is reasonable because it has been proved 

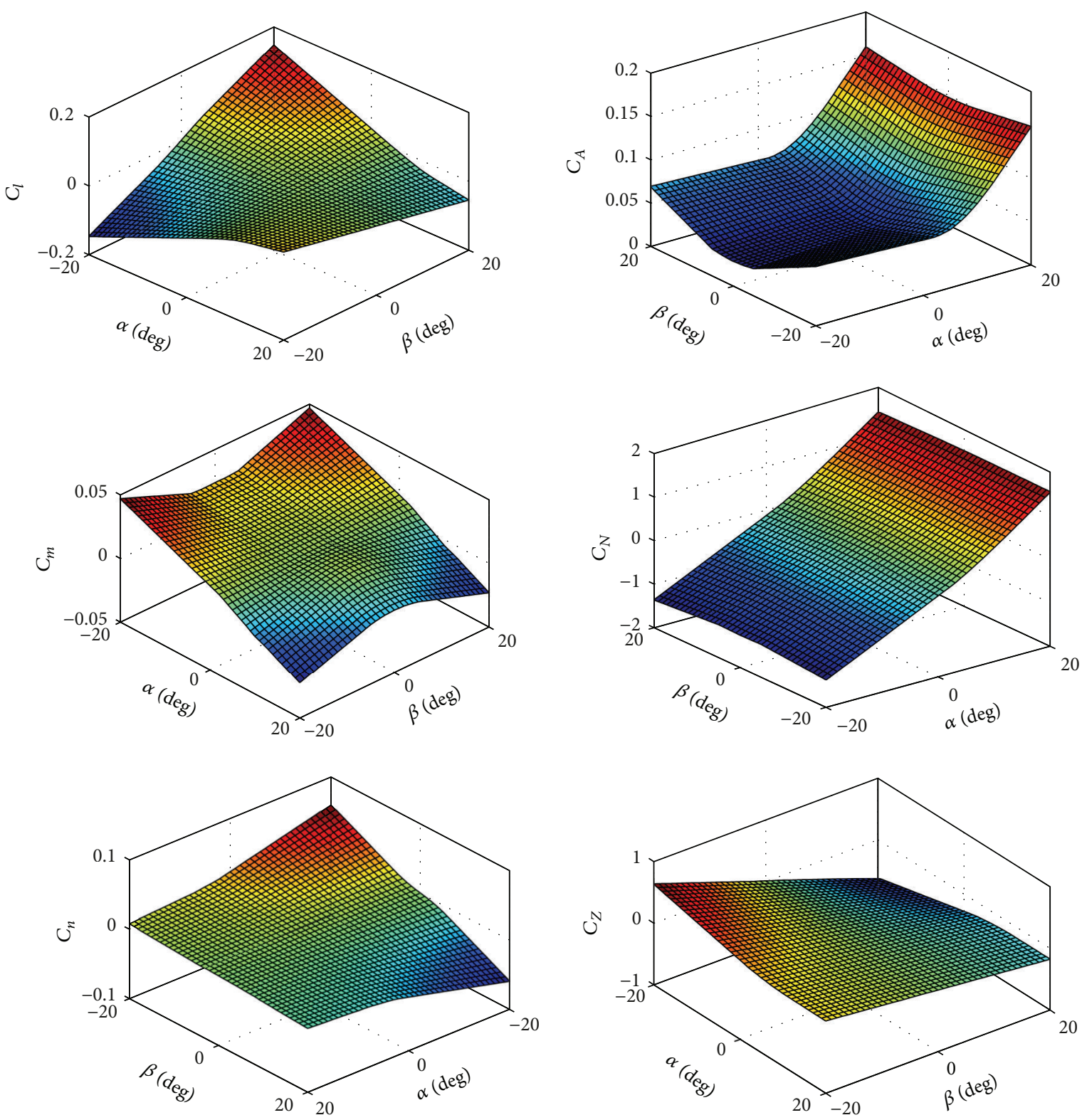

(a) Moment coefficients

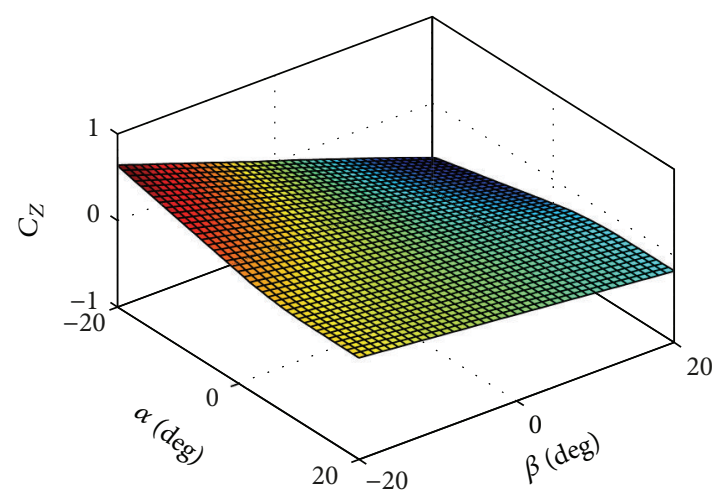

(b) Force coefficients

Figure 1: Moment and force coefficients at $\delta_{r}=\delta_{l}=0.1 \mathrm{deg}$.

that the observation error is uniformly ultimately bounded (UUB, $\in L_{\infty}$ ) within a region of arbitrarily small size [14]. Thus, we will neglect the observation error in the following parts.

The estimation of $\widehat{\Pi}_{i}(i=1,2)$ plays an extremely important role in the whole control system. The total disturbance should be estimated accurately online, and fuzzy theory is quite suitable for this condition. After choosing the membership functions, the fuzzy disturbance observer will have different response time and convergence speed in accordance with which membership area the observation error is located in. This kind of intelligent observer will make the online identification process achieve excellent performance.

\section{Disturbance Observer Based on Fuzzy Theory}

3.1. Introduction of Extended State. Nonlinear extended state observer (NESO) performs well in not only observing all the system states but also monitoring the uncertainties and external disturbance. Under this framework, NESO can derive various forms due to different nonlinear functions involved. In this paper, fuzzy theory is introduced to deal with disturbance estimation error. The observer behavior will be automatically adjusted by the error level, which makes the observer achieve perfect transient response. According to 


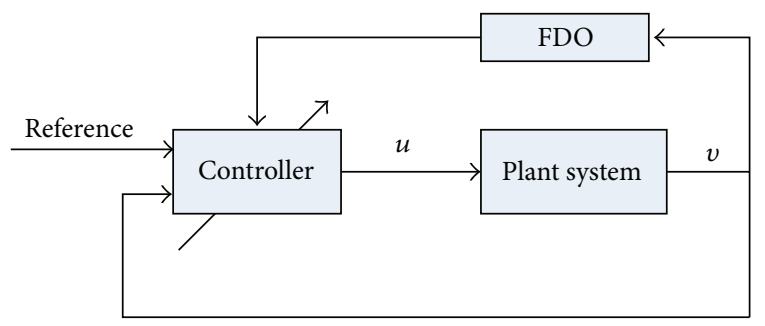

FIGURE 2: Controlled system based on FDO.

the system (8), the following NESO is designed to estimate the total disturbance:

$$
\begin{aligned}
& \Delta \boldsymbol{\Gamma}=\boldsymbol{\varrho}-\boldsymbol{\Gamma}=\left[\begin{array}{llllll}
\Delta \alpha & \Delta \beta & \Delta \gamma & \Delta \omega_{x} & \Delta \omega_{y} & \Delta \omega_{z}
\end{array}\right]^{T} \\
& \dot{\boldsymbol{Q}}=\mathbf{K}(\boldsymbol{\Theta}) \mathbf{U}+\widehat{\Pi}-\mathrm{c} \Delta \boldsymbol{\Gamma} \\
& \widehat{\Pi}=\mathbf{G}(\boldsymbol{\Gamma}, \mathbf{u}),
\end{aligned}
$$

where

$$
\begin{aligned}
& \boldsymbol{\Delta} \boldsymbol{\Gamma}=\left[\boldsymbol{\Delta} \boldsymbol{\Theta}^{T} \boldsymbol{\Delta} \boldsymbol{\Omega}^{T}\right]^{T}: \text { reconfigured states error, } \\
& \varrho=\left[\begin{array}{llllll}
\alpha^{\prime} & \beta^{\prime} & \gamma^{\prime} & \omega_{x}^{\prime} & \omega_{y}^{\prime} & \omega_{z}^{\prime}
\end{array}\right]^{T} \text { : reconfigured states, } \\
& \widehat{\boldsymbol{\Pi}}=\left[\widehat{\boldsymbol{\Pi}}_{1}^{T} \widehat{\boldsymbol{\Pi}}_{2}^{T}\right]^{T} \text { : estimated total disturbance. }
\end{aligned}
$$

In system (13), the nonlinear function $\mathbf{G}(\boldsymbol{\Gamma}, \mathbf{u})$ is a fuzzy inference engine, which is used to estimate the total disturbance. $\widehat{\Pi}$ is the extended state, which is defined in the subtitle. The $\mathbf{G}(\boldsymbol{\Gamma}, \mathbf{u})$ can be also rewritten as $\mathbf{G}(\boldsymbol{\Gamma})$ because the input $\mathbf{u}(\Gamma)$ is determined by full-state feedback. The constant vector c, if chosen properly, will control the weight the reconfigured error has in the observer equation and it will enhance the stability of the whole system by negative feedback. The goal for this observer is to make the following equation hold:

$$
\lim _{t \rightarrow+\infty}\|\boldsymbol{\varrho}-\boldsymbol{\Gamma}\|=\mathbf{0} .
$$

Figure 2 depicts the whole controlled system based on fuzzy disturbance observer (FDO).

3.2. Disturbance Observer Based on Fuzzy Theory. In [14], Euntai gave detailed explanations on fuzzy disturbance observer. A typical fuzzy system follows the IF-THEN rules which are characterized by a set of condition $\rightarrow$ action rules, or in IF-THEN form as follows [22]:

IF premise THEN consequent,

or

$$
\text { IF } v_{1} \text { is } A_{1}^{i} \text { and, ..., and } v_{n} \text { is } A_{n}^{i} \text { THEN } y^{i} \text { is } B \text {. }
$$

In this paper, the principle of product inference, centeraverage and singleton fuzzifier is adopted. Then, the expression of the estimated total disturbance is obtained as follows:

$$
\mathbf{G}(\boldsymbol{\Gamma})=\frac{\sum_{i=1}^{m} y^{i}\left(\prod_{j=1}^{n} \mu_{A_{j}^{i}}\left(\Gamma_{j}\right)\right)}{\sum_{i=1}^{m}\left(\prod_{j=1}^{n} \mu_{A_{j}^{i}}\left(\Gamma_{j}\right)\right)}=\widehat{\theta}^{T} \xi(\boldsymbol{\Gamma}),
$$

where $\hat{\theta} \in \mathbb{R}^{27 \times 1}, \xi(\boldsymbol{\Gamma}) \in \mathbb{R}^{27 \times 1} . \widehat{\theta}$ is the adjustable vector, which is composed of $y^{i}$, and $\xi(\boldsymbol{\Gamma})$ is the fuzzy basis function. Equation (18) is a general solution formula for every channel of the estimated disturbance vector $\widehat{\Pi}$. The adaptive parameter is shown as follows (take the channel of $\alpha$, e.g.):

$$
\dot{\hat{\theta}}_{\alpha}=\lambda_{\alpha} \Delta \alpha \xi(\alpha, \beta, \gamma)
$$

and the fuzzy basis functions are as follows:

$$
\xi_{i}=\frac{\prod_{j=1}^{3} \mu_{A_{j}^{i}}\left(\Gamma_{j}\right)}{\sum_{i=1}^{3}\left(\prod_{j=1}^{3} \mu_{A_{j}^{i}}\left(\Gamma_{j}\right)\right)},
$$

where $\left[\begin{array}{lll}\Gamma_{1} & \Gamma_{2} & \Gamma_{3}\end{array}\right]=[\alpha \beta \gamma], \xi(\alpha, \beta, \gamma)=\left[\xi_{1}, \xi_{2}, \ldots, \xi_{27}\right]^{T}$, and

$$
\begin{aligned}
& \mu_{A_{1}^{1}}\left(\Gamma_{1}\right)=\frac{1}{1+\exp \left(2\left(\Gamma_{1}+0.6\right)\right)}, \\
& \mu_{A_{1}^{2}}\left(\Gamma_{1}\right)=\exp \left(-\left(\Gamma_{1}\right)^{2}\right), \\
& \mu_{A_{1}^{3}}\left(\Gamma_{1}\right)=\frac{1}{1+\exp \left(-2\left(\Gamma_{1}-0.6\right)\right)}, \\
& \mu_{A_{2}^{1}}\left(\Gamma_{2}\right)=\frac{1}{1+\exp \left(2\left(\Gamma_{2}+0.1\right)\right)}, \\
& \mu_{A_{2}^{2}}\left(\Gamma_{2}\right)=\exp \left(-\left(\Gamma_{2}\right)^{2}\right), \\
& \mu_{A_{2}^{3}}\left(\Gamma_{2}\right)=\frac{1}{1+\exp \left(-2\left(\Gamma_{2}-0.1\right)\right)}, \\
& \mu_{A_{3}^{1}}\left(\Gamma_{3}\right)=\frac{1}{1+\exp \left(2\left(\Gamma_{3}+0.9\right)\right)}, \\
& \mu_{A_{3}^{2}}\left(\Gamma_{3}\right)=\exp \left(-\left(\Gamma_{3}\right)^{2}\right), \\
& \mu_{A_{3}^{3}}\left(\Gamma_{3}\right)=\frac{1}{1+\exp \left(-2\left(\Gamma_{3}-0.9\right)\right)} .
\end{aligned}
$$

For the sake of reducing calculation amount, there are only three fuzzy membership functions for each channel of $\alpha, \beta$, and $\gamma$, which are shown in Figure 3 .

The disturbance observation error $\Delta \Gamma$ will be uniformly ultimately bounded $\left(\Delta \Gamma \in L_{\infty}\right)$, which has been proved in [14]. And this method will accelerate the convergence speed of the FDO when the observation error is approaching zero. As for (19), the fuzzy basis functions $\xi(\alpha, \beta, \gamma)$ will be the same when dealing with the channels of $\alpha, \beta$, and $\gamma$. But when estimating the total disturbance of the channels of $\omega_{x}, \omega_{y}$, and $\omega_{z}$, the functions will change to $\xi\left(\omega_{x}, \omega_{y}, \omega_{z}\right)$, and the method of obtaining it is similar to the former. 

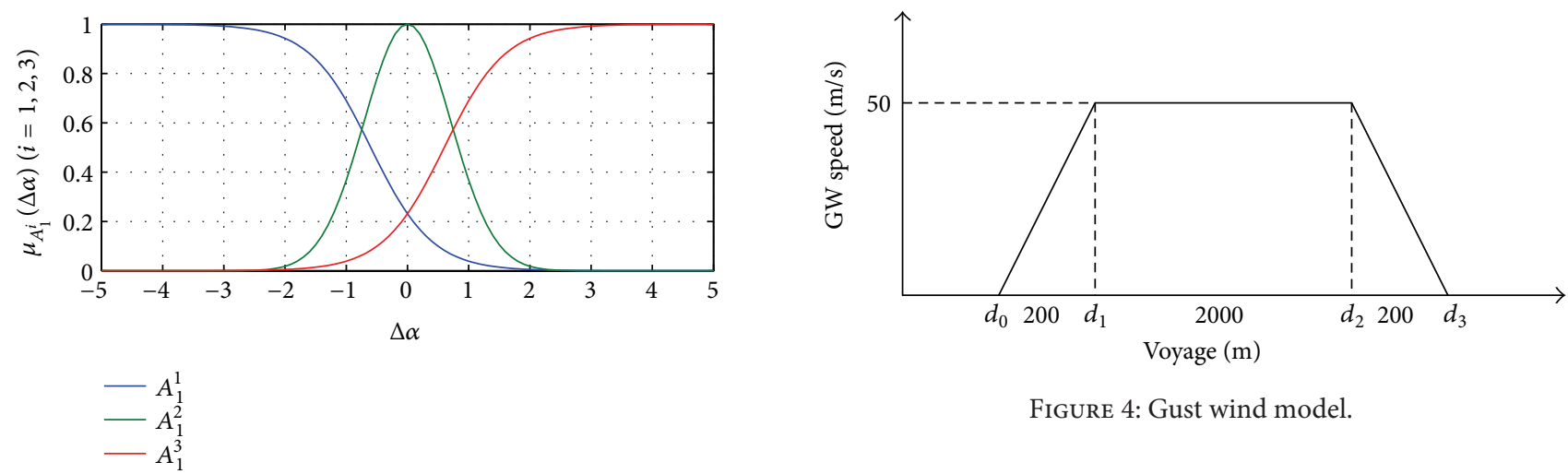

Figure 4: Gust wind model.
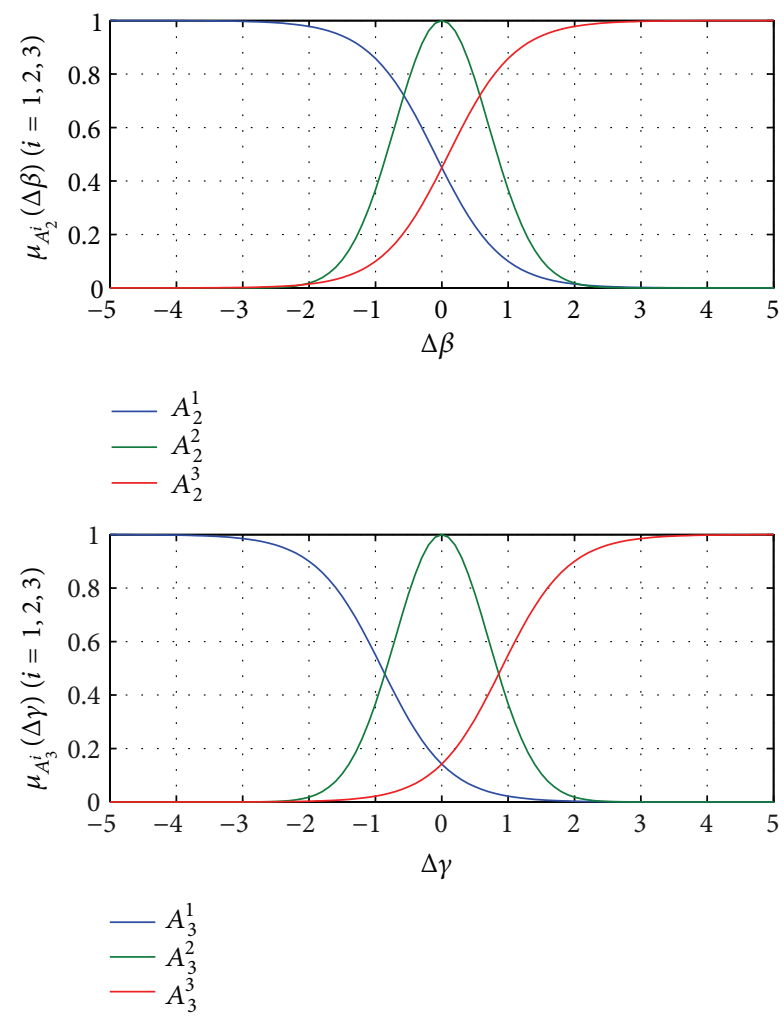

TABLE 1: Distractions of parameters in the plant system model.

\begin{tabular}{lccc}
\hline Parameter & Distraction & Parameter & Distraction \\
\hline$\Delta C_{A}$ & $20 \% C_{A}$ & $\Delta C_{N}$ & $15 \% C_{N}$ \\
$\Delta C_{Z}$ & $20 \% C_{Z}$ & $\Delta C_{l}$ & $40 \% C_{l}+0.001$ \\
$\Delta C_{n}$ & $40 \% C_{n}+0.002$ & $\Delta C_{m}$ & $30 \% C_{m}+0.001$ \\
$\Delta C_{l p}$ & $50 \% C_{l p}$ & $\Delta C_{n r}$ & $50 \% C_{n r}$ \\
$\Delta C_{m q}$ & $50 \% C_{m q}$ & $\Delta m$ & $20 \% m$ \\
$\Delta J_{X}$ & $8 \% J_{X}$ & $\Delta J_{Y}$ & $8 \% J_{Y}$ \\
$\Delta J_{Z}$ & $8 \% J_{Z}$ & $\Delta J_{X Y}$ & $15 \% J_{X Y}$ \\
$\Delta J_{X Z}$ & 0.4 & $\Delta J_{Y Z}$ & 0.4 \\
\hline
\end{tabular}

TABLE 2: Relationship between stationary wind speed and flight altitude.

\begin{tabular}{lccc}
\hline Altitude $(\mathrm{km})$ & $f_{w}^{S W}(\mathrm{~m} / \mathrm{s})$ & Altitude $(\mathrm{km})$ & $f_{w}^{S W}(\mathrm{~m} / \mathrm{s})$ \\
\hline 35.0 & 64.0 & 64.5 & 101.7 \\
39.0 & 64.0 & 65.0 & 101.0 \\
44.0 & 84.4 & 69.0 & 95.4 \\
49.0 & 112.4 & 69.5 & 94.1 \\
59.0 & 112.4 & 70.0 & 92.8 \\
59.5 & 111.4 & 74.0 & 82.4 \\
60.0 & 110.4 & 107.0 & 82.4 \\
64.0 & 102.4 & 110.0 & 80.5 \\
\hline
\end{tabular}

FIgURE 3: Fuzzy basis functions for $\alpha, \beta$, and $\gamma$.

Given $\mathbf{K}=\left[\begin{array}{ll}k_{i 1} & k_{i 2}\end{array}\right], \mathbf{E}=\left[\begin{array}{ll}\mathbf{e}_{i} & \dot{\mathbf{e}}_{i}\end{array}\right]^{T}$, and $\mathbf{u}_{\mathbf{c}}=$ $\left[u_{c 1}, u_{c 2}, u_{c 3}\right]^{T}, i=1,2,3$, we set the following sliding-mode surface function for any one of the channels:

$$
\begin{gathered}
\mathbf{s}_{i}=\mathbf{K E}=k_{i 1}\left(\boldsymbol{\Theta}_{i}^{*}-\boldsymbol{\Theta}_{i}\right)+k_{i 2}\left(\boldsymbol{\Omega}_{i}^{*}-\boldsymbol{\Omega}_{i}\right), \\
\dot{\mathbf{s}}_{i}=k_{i 1}\left(\dot{\boldsymbol{\Theta}}_{i}^{*}-\boldsymbol{\Omega}_{i}\right)+k_{i 2}\left(\dot{\boldsymbol{\Omega}}_{i}^{*}-u_{c i}\right)=\operatorname{slaw}_{i},
\end{gathered}
$$

After observing and removing the total disturbance, the cascade system (12) is obtained. In this paper, the slidingmode method is used to deal with the cascade system. Angle tracking errors are defined as follows:

$$
\mathbf{e}=\Theta^{*}-\boldsymbol{\Theta}
$$

where $\Theta^{*}$ is the given reference trajectory. Differentiating (22),

$$
\dot{\mathbf{e}}=\dot{\mathbf{\Omega}}^{*}-\dot{\boldsymbol{\Theta}}=\mathbf{\Omega}^{*}-\mathbf{\Omega}
$$

where slaw $_{i}$ is the sliding-mode approaching law. In order to achieve good tracking performance, in this paper, we choose the exponential approaching law instead of the traditional proportional approaching law:

$$
\operatorname{slaw}_{i}=-\varepsilon_{i} \operatorname{sgn}\left(\mathbf{s}_{i}\right)-\beta_{i} \mathbf{s}_{i} .
$$

In (24), the sliding-mode surface function $\mathbf{s}_{i}$ satisfies threes conditions: (1) $\mathbf{s}_{i}\left(t=t_{0}\right)=0$, (2) $\mathbf{s}_{i}$ is differentiable, and (3) $\mathbf{s}_{i}^{T} \dot{\mathbf{s}}_{i}<0$. These conditions guarantee not only the stability of tracking process but also the steady tracking error 

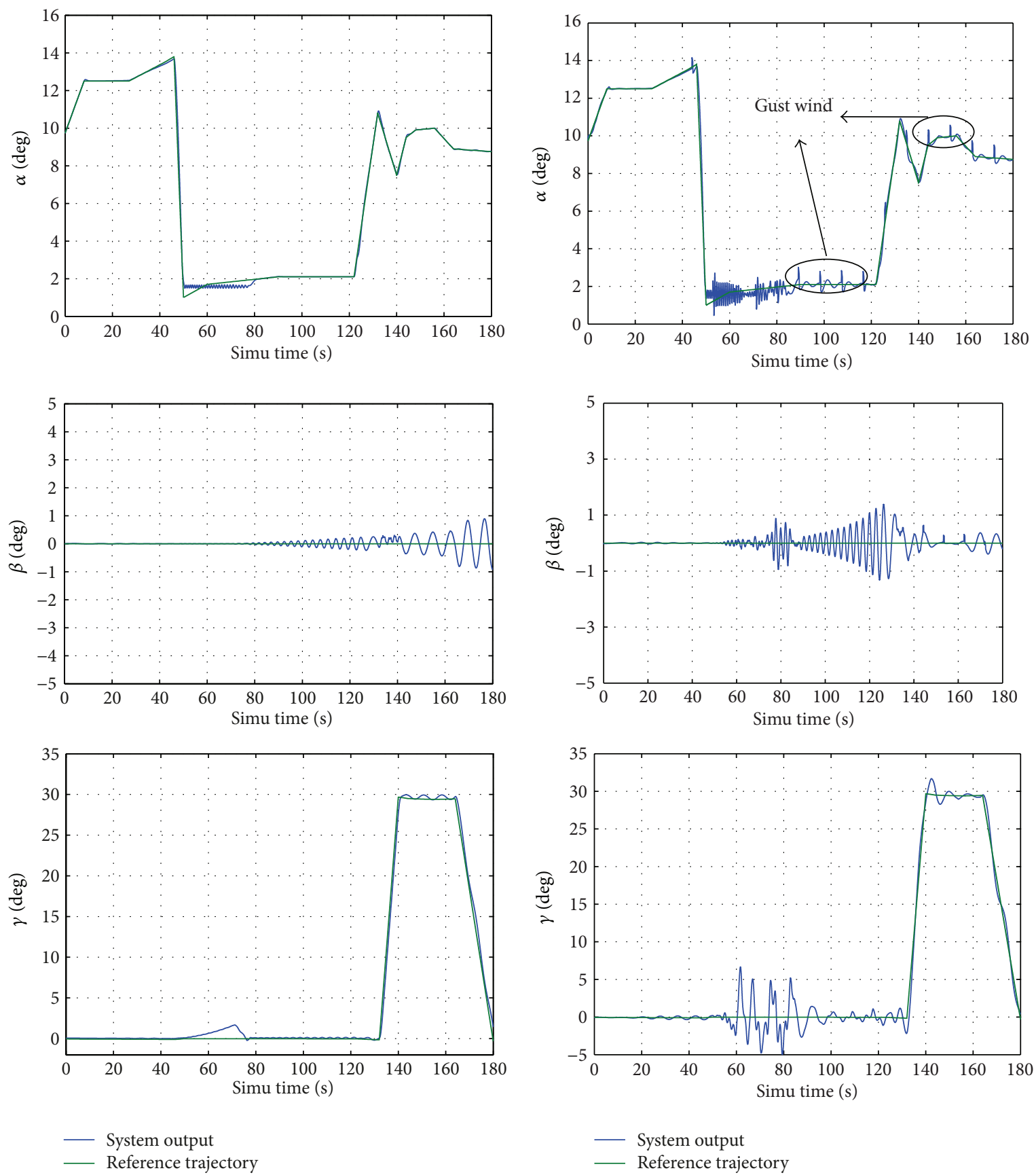

(a) $\alpha, \beta$, and $\gamma$ performance in tracking references without total disturbance

(b) $\alpha, \beta$, and $\gamma$ performance in tracking references with total disturbance

FIgURE 5: Tracking results without and with total disturbance.

asymptotically approaching to zero. Substituting (25) into (24), we get the controlled input as

$$
u_{c i}=\frac{1}{k_{i 2}}\left(k_{i 1}\left(\dot{\Theta}_{i}^{*}-\mathbf{\Omega}_{i}\right)+\varepsilon_{i} \operatorname{sgn}\left(\mathbf{s}_{i}\right)+\beta_{i} \mathbf{s}_{i}\right)+\dot{\boldsymbol{\Omega}}_{i}^{*} .
$$

Remark. According to the repeated trials, it is more likely to achieve good control performance when the parameters are chosen as $\varepsilon_{1}=5, \beta_{1}=10, k_{11}=3$, and $k_{12}=1$. All of these parameters can significantly influence the stability, the rising time and the tracking error of this closed-loop system but no single parameter can determine any of them independently. Thus, so far the adjusting method is one of the best methods to determine these parameters. Substituting (26) into (11), we obtain the final controller expression with the negative feedback of the integral of the tracking error:

$$
\mathbf{u}=\mathbf{B}^{\dagger}(\boldsymbol{\Theta})\left(-\widehat{\Pi}_{2}+\left[u_{c 1}, u_{c 2}, u_{c 3}\right]^{T}-\mathbf{K}_{a} \int \mathbf{e}\right),
$$

where

$$
\mathbf{K}_{a}>\mathbf{0}
$$



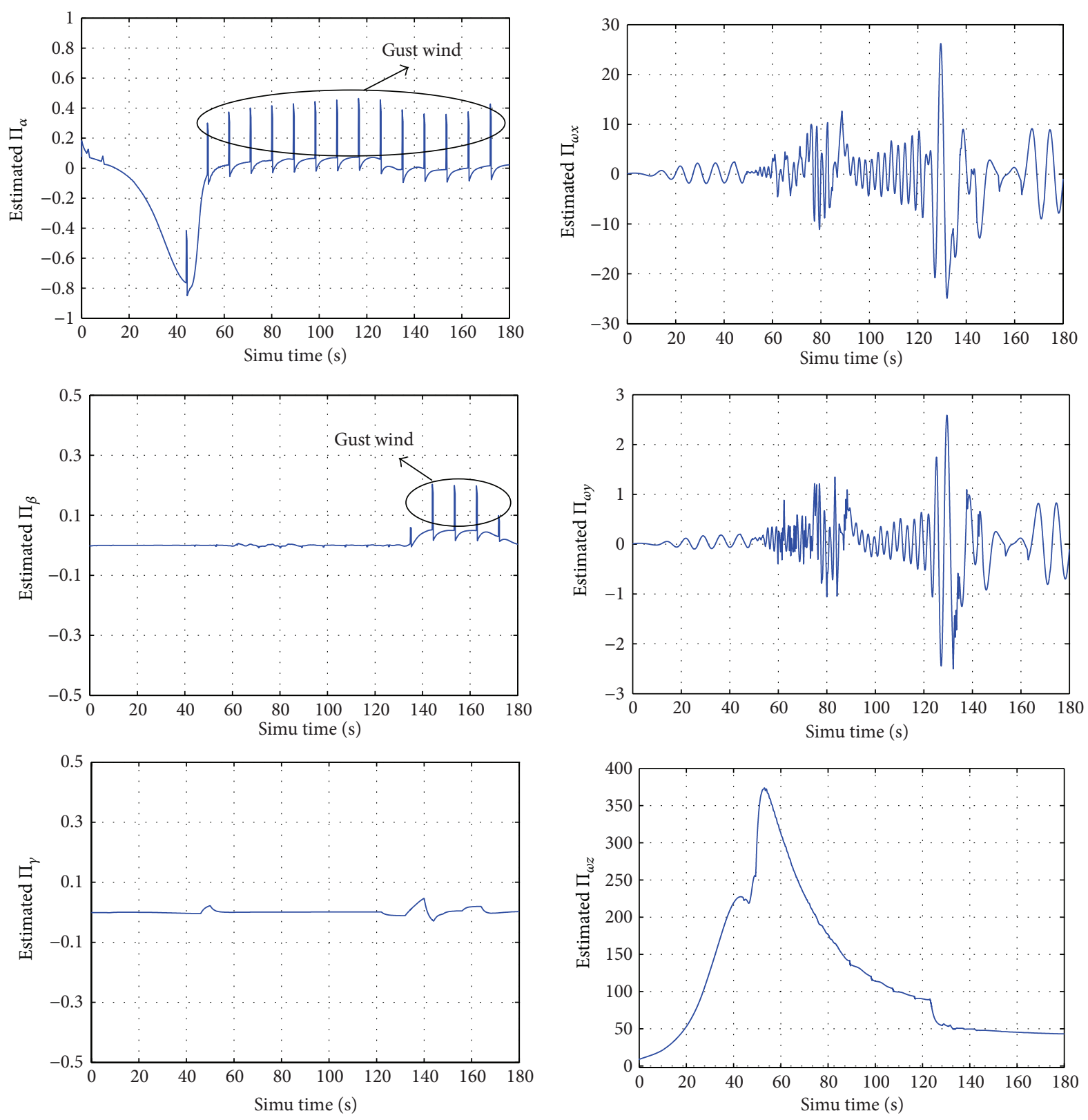

(a) Estimated disturbance of angle channels

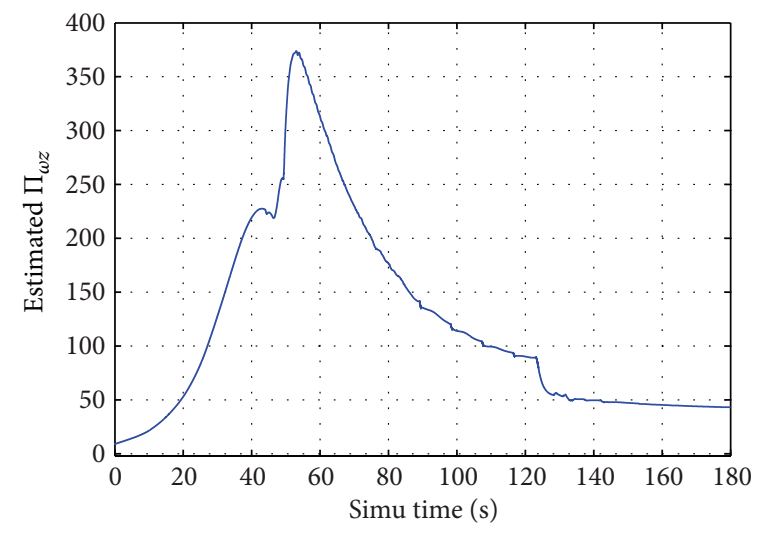

(b) Estimated disturbance of angle-rate channels

FIgURE 6: Total disturbance estimations.

\section{Simulations}

In this part, a wide range of parameter uncertainties and external disturbance is set for the plant system, and parameter uncertainties are realized by the distractions of relevant parameters in the model of plant system. The goal is to test the ability of the controlled system to resist disturbance and its own uncertainties. It is also expected that the disturbance observer will achieve good performance.

5.1. Parameters Distractions and External Wind Disturbance. In Table 1, all of the distracted parameters are listed, in which numbers expressed in percentage mean relative distractions, while others indicate absolute distractions. All of these changes occur in the model of plant system, which represents the vehicle in the virtual reality environment for flight.

Then, the model for wind disturbance is given as follows. In general, both stationary wind and gust wind will not be taken into consideration until the simulation runs to 60 seconds. The speed of stationary wind will change according to the flight altitude, and the accurate value for this speed can be gotten by linear interpolation. Table 2 demonstrates the relationship between the wind speed and the flight altitude.

As for the gust wind model, a regular model with the period of $50 \mathrm{~km}$ is considered, which is illustrated in Figure 4. For each time, the gust wind starts at $d_{0}$ and ends at $d_{3}$. After 
$50 \mathrm{~km}$ flight, which is at $d_{0}+50 \mathrm{~km}$, the same wind reappears again.

The directions of both stationary wind and gust wind are stochastic, and $A_{w}$ denotes the azimuth angle of wind. Thus, the wind vector is as follows:

$$
\vec{V}_{w}=\vec{V}_{\mathrm{SW}}+\vec{V}_{\mathrm{GW}},
$$

where

$$
\begin{gathered}
\vec{V}_{\mathrm{SW}}=f_{w}^{\mathrm{SW}} \times\left[\begin{array}{c}
\cos \left(A_{w}\right) \\
0 \\
-\sin \left(A_{w}\right)
\end{array}\right], \\
\vec{V}_{\mathrm{GW}}=\sqrt{2} \times f_{w}^{\mathrm{GW}} \times\left[\begin{array}{c}
\cos \left(A_{w}\right) \\
0 \\
-\sin \left(A_{w}\right)
\end{array}\right]+f_{w}^{\mathrm{GW}} \times\left[\begin{array}{c}
0 \\
-1 \\
0
\end{array}\right] .
\end{gathered}
$$

The wind vector mainly influences the system outputs ( $\alpha$ and $\beta$ ) by changing the speed and direction of the incoming flow. So far, all the modeling work for total disturbance has been finished. In the next subsection, several pictures of simulation results will be shown.

5.2. Simulation Results. The main goal for this paper is to make the HFV track reference trajectory with high performance in severe situation. At first, it only tests the performance of the controller based on sliding-mode method; thus, total disturbance is not added into the plant system. Simulation results are shown in Figure 5(a). It can be clearly learned that the controller has performed an excellent work for the tracking task. Although the tracking performance is not good at about 50 seconds because the angle of attack suddenly drops which results in the rapid declination of lift force, the whole system soon goes back to its stable states under the action of controller and the error is limited within two degrees.

When the total disturbance is added into the system, the performance of the controlled system is shown in Figure 5(b). From these figures, it demonstrates that even though the disturbance exerts negative impact (marked in the figures) on the whole system, it has successfully finished the tracking task and left minor steady error. All these accomplishments benefit from the excellent performance of FDO. Figure 6 demonstrates that the total disturbance is superbly monitored by the FDO. In Figure 6(a), it clearly shows that the periodic gust wind disturbance is totally observed, which is in accordance with the real situation.

\section{Conclusions}

This study indicates that, although the flight condition for the hypersonic flight vehicle contains mass uncertainties and gust wind disturbance, the fuzzy disturbance observer monitors the total disturbance very well. The definition of total disturbance provides a new way of disentangling complex structure information. This method contains both the analytical dynamic inversion and the numerical online identification, which contributes to its excellent performance when the tracking trajectory varies drastically. In the future, this work is expected to focus on the study of the estimated total disturbance separation and analysis.

\section{Acknowledgments}

This work is supported by the National Natural Science Key Foundation of China under Grants nos. 91216124, 91116002, 90916003, 91216304, and 11332001; Foundation of national astronautica key lab (2012-865-6).

\section{References}

[1] D. K. Schmidt, "Optimum mission performance and multivariable flight guidance for airbreathing launch vehicles," Journal of Guidance, Control, and Dynamics, vol. 20, no. 6, pp. 1157-1164, 1997.

[2] F. R. Chavez and D. K. Schmidt, "Analytical aeropropulsive/aeroelastic hypersonic-vehicle model with dynamic analysis," Journal of Guidance, Control, and Dynamics, vol. 17, no. 6, pp. 1308-1319, 1994.

[3] M. A. Bolender and D. B. Doman, "A nonlinear longitudinal dynamical model of an air-breathing hypersonic vehicle," Journal of Spacecraft and Rockets, vol. 44, no. 2, pp. 374-387, 2007.

[4] D. Sigthorsson, P. Jankovsky, A. Serrani, S. Yurkovich, M. A. Bolender, and D. B. Doman, "Robust linear output feedback control of an airbreathing hypersonic vehicle," Journal of Guidance, Control, and Dynamics, vol. 31, no. 4, pp. 1052-1066, 2008.

[5] J. T. Parker, A. Serrani, S. Yurkovich, M. A. Bolender, and D. B. Doman, "Control-oriented modeling of an air-breathing hypersonic vehicle," Journal of Guidance, Control, and Dynamics, vol. 30, no. 3, pp. 856-869, 2007.

[6] J. McNamara and P. Friedmann, "Aeroelastic and aerothermoelastic analysis of hypersonic vehicles: current status and future trends," in Proceedings of the 48th AIAA/ASME/ASCE/AHS/ASC Structures, Structural Dynamics, and Materials Conference, pp. 3814-3868, Honolulu, Hawaii, USA, April 2007, AIAA paper no. 2007-2013.

[7] R. Lind, "Linear parameter-varying modeling and control of structural dynamics with aerothermoelastic effects," Journal of Guidance, Control, and Dynamics, vol. 25, no. 4, pp. 733-739, 2002.

[8] L. Fiorentini, A. Serrani, M. A. Bolender, and D. B. Doman, "Nonlinear robust adaptive control of flexible air-breathing hypersonic vehicles," Journal of Guidance, Control, and Dynamics, vol. 32, no. 2, pp. 401-416, 2009.

[9] L. Fiorentini, A. Serrani, M. A. Bolender, and D. B. Doman, "Nonlinear robust/adaptive controller design for an airbreathing hypersonic vehicle model," in Proceedings of the AIAA Guidance, Navigation, and Control Conferenc and Exhibit, pp. 269-284, Hilton Head, SC, USA, August 2007, AIAA paper no. 2007-6329.

[10] H. Xu, M. Mirmirani, and P. Ioannou, "Adaptive sliding mode control design for a hypersonic flight vehicle," Journal of Guidance, Control, and Dynamics, vol. 27, no. 5, pp. 829-838, 2004.

[11] Q. Wang and R. F. Stengel, "Robust nonlinear control of a hypersonic aircraft," Journal of Guidance, Control, and Dynamics, vol. 23 , no. 4, pp. 577-585, 2000.

[12] H. Buschek and A. J. Calise, "Uncertainty modeling and fixedorder controller design for a hypersonic vehicle model," Journal 
of Guidance, Control, and Dynamics, vol. 20, no. 1, pp. 42-48, 1997.

[13] H. Li, Y. Si, L. Wu, X. Huang, and H. Gao, "Guaranteed cost control with poles assignment for a flexible air-breathing hypersonic vehicle," International Journal of Systems Science, vol. 42, no. 5, pp. 863-876, 2011.

[14] K. Euntai, "A fuzzy disturbance observer and its application to control," IEEE Transactions on Fuzzy Systems, vol. 10, no. 1, pp. 77-84, 2002.

[15] D. Ye and G. Yang, "Adaptive fault-tolerant tracking control against actuator faults with application to flight control," IEEE Transactions on Control Systems Technology, vol. 14, no. 6, pp. 1088-1096, 2006.

[16] H. Dong, Z. Wang, and H. Gao, "Fault detection for Markovian jump systems with sensor saturations and randomly varying nonlinearities," IEEE Transactions on Circuits and Systems, vol. 59, no. 10, pp. 2354-2362, 2012.

[17] J. Hu, Z. Wang, H. Gao, and L. K. Stergioulas, "Extended Kalman filtering with stochastic nonlinearities and multiple missing measurements," Automatica, vol. 48, no. 9, pp. 20072015, 2012.

[18] C. Marrison and R. Stengel, "Design of robust control systems for a hypersonic aircraft," Journal of Guidance, Control, and Dynamics, vol. 21, no. 1, pp. 58-63, 1998.

[19] D. Ding, Z. Wang, H. Dong, and H. Shu, "Distributed H1 state estimation with stochastic parameters and nonlinearities through sensor networks: the finite-horizon case," Automatica, vol. 48, no. 8, pp. 1575-1585, 2012.

[20] D. Ding, Z. Wang, B. Shen, and H. Shu, "H1 state estimation for discrete-time complex networks with randomly occurring sensor saturations and randomly varying sensor delays," IEEE Transactions on Neural Networks and Learning Systems, vol. 23, no. 5, pp. 725-736, 2012.

[21] J. Han, "From PID to active disturbance rejection control," IEEE Transactions on Industrial Electronics, vol. 56, no. 3, pp. 900906, 2009.

[22] M. P. Kevin and S. Yurkovich, Fuzzy Control, Addison-Wesley, Reading, Mass, USA, 1997. 


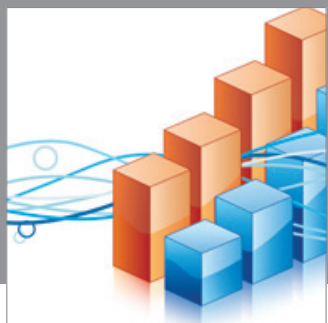

Advances in

Operations Research

mansans

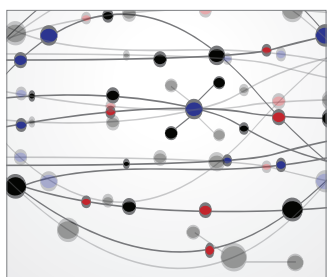

The Scientific World Journal
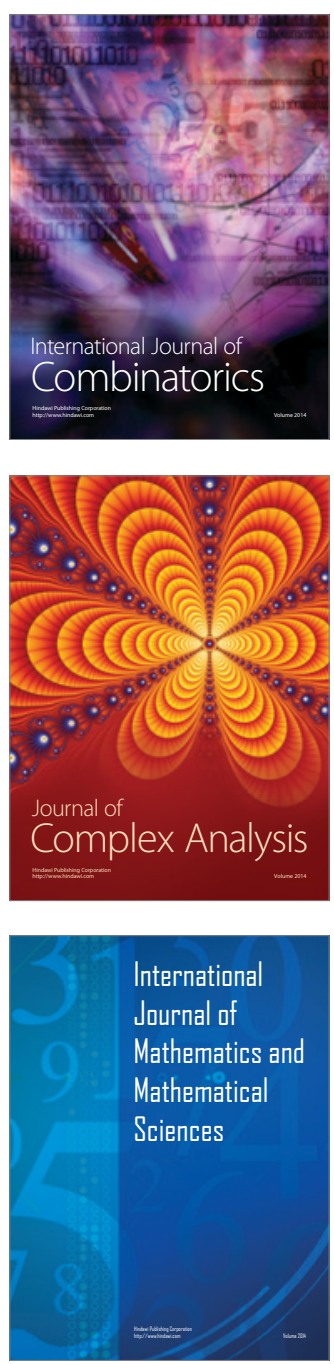
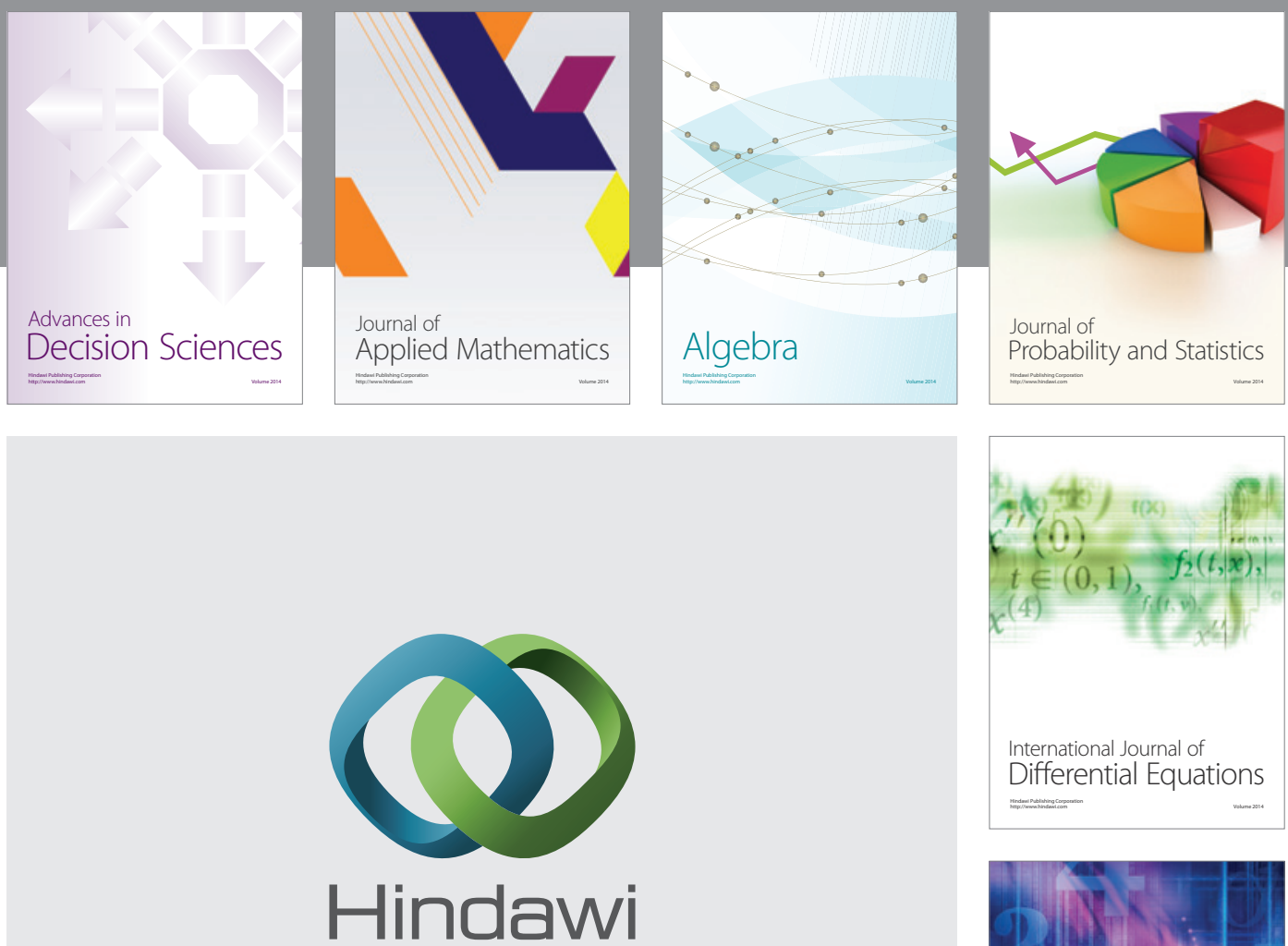

Submit your manuscripts at http://www.hindawi.com
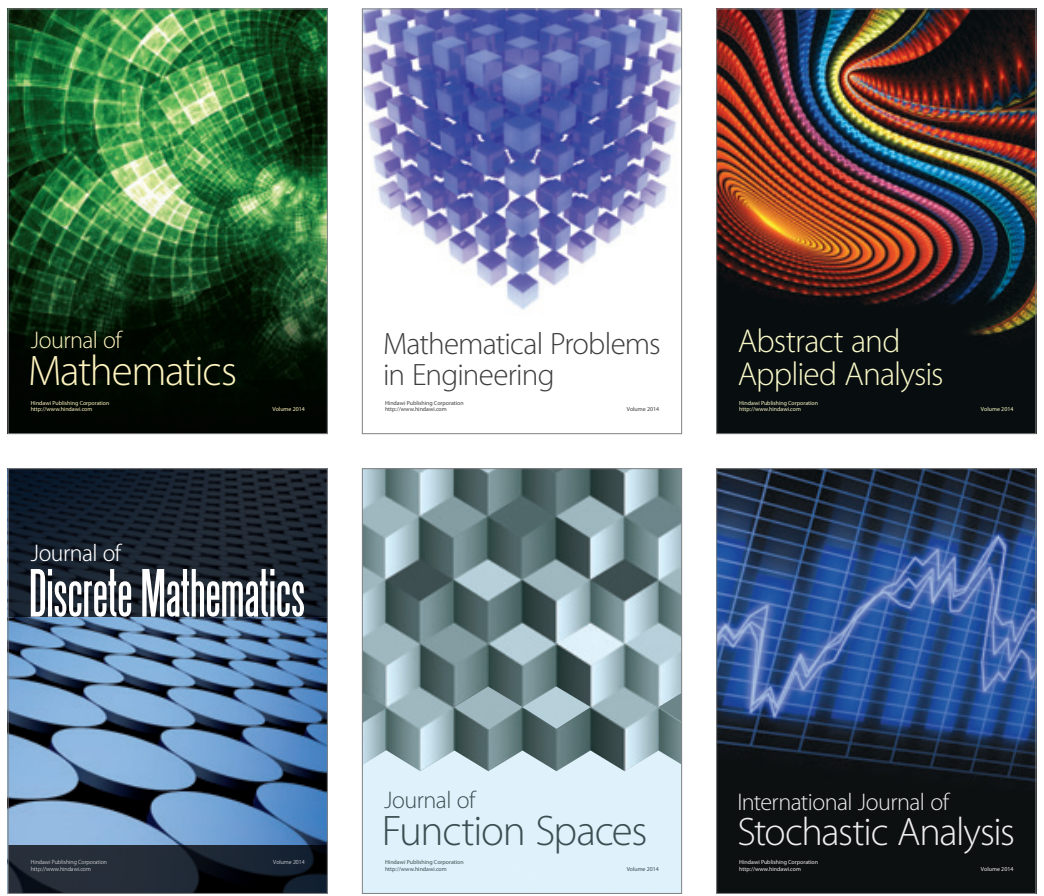

Journal of

Function Spaces

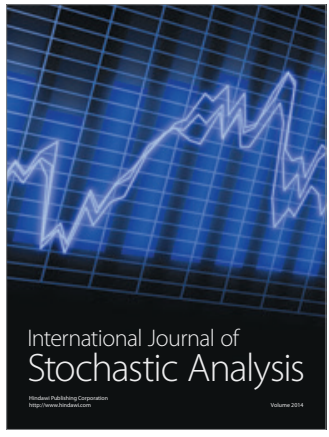

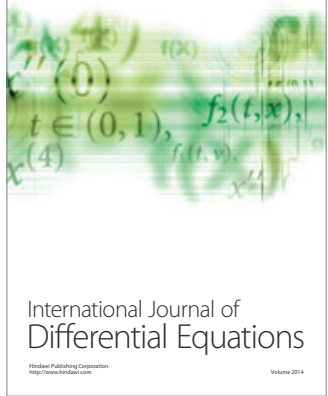
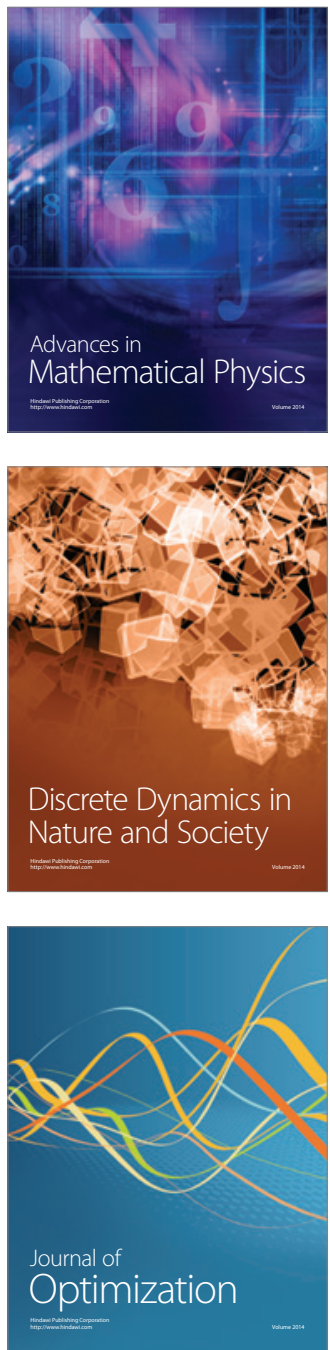